\title{
The Nucleon Resonance Program at JLab
}

\author{
Ralf W. Gothe for JLab and the CLAS Collaboration \\ Department of Physics and Astronomy, University of South Carolina, Columbia, SC 29208
}

\begin{abstract}
The status of the program to study baryon resonances at Jefferson Lab will be exemplified by the latest results on resonance parameters and transition form factors in single and double-pion production as well as kaon-hyperon decays.
\end{abstract}

Keywords: nucleon resonances and transition form factors.

PACS: 13.60.Rj, 13.60.-r, 14.20.-c, 14.20.Gk.

\section{INTRODUCTION}

Two of the four fundamental forces, the weak and especially the electromagnetic one, are reasonably well understood within the underlying framework of perturbative quantum field theories. A complete quantum field theoretical description of the gravitational force is still in its early stages and experimentally the graviton, the field carrier itself, has not yet been measured. In the case of the strong force, we have two experimentally verified perturbative quantum field theories; perturbative Quantum Chromodynamics (pQCD) at small distances which is governed by gluon fields and Chiral Perturbation Theory (ChPT) at larger distances which is governed by pion fields. However, the strong field at intermediate distances, where it generates more than $98 \%$ of the total mass of all normal matter, is not understood on similar firm grounds. Laboratories currently provide complementary hadronic or electromagnetic probes in the best suited energy range to perform precision experiments that test the nature of the strong force in this intermediate confinement regime. One of the leading laboratories in this research field is Jefferson Lab (JLab), where some of the most pressing experiments are planned and carried out. Examples reported here will focus on: transition form factor measurements at low $\mathrm{Q}^{2}$ to investigate the pion fields and to bridge the gap to ChPT, and at high $\mathrm{Q}^{2}$ to investigate the transition to $\mathrm{pQCD}$ in exclusive channels; as well as searching for resonances in sequential decays and for resonances that do not couple to single-pion nucleon states to test the quark flavor symmetry or for highly excited resonances to test chiral symmetry restoration; and pinning down resonance parameters by precision measurements of polarization and double-polarization observables in single- and double-meson production channels.

\section{SINGLE-MESON PRODUCTION}

A large portion of the nuclear physics community enthusiastically started to investigate baryon resonances as new optimized detector systems with large solid angle and momentum coverage like CLAS and new high-intensity continuous electron 
beams like at JLab became available. The high versatility of the provided electromagnetic probes that have merely any initial state interaction has been intriguing ever since. The isoscalar or isovector and the electric or magnetic or longitudinal character of the coupling to hadronic matter probe different aspects of the strong interaction; but soon after scientists had to experience the annoying complications introduced by the desired versatility of the electromagnetic probe that mixes all the different coupling amplitudes simultaneously into the measured cross sections. The way out has been successfully demonstrated in the case of the $\mathrm{N}$ to $\Delta(1232)$ transition where the small resonant electric (EMR) and scalar quadrupole (SMR) amplitudes could be extracted with respect to the dominant magnetic dipole amplitude at intermediate momentum transfers $\left(0.2(\mathrm{GeV} / \mathrm{c})^{2}<\mathrm{Q}^{2}<1.0(\mathrm{GeV} / \mathrm{c})^{2}\right)$ with absolute systematic uncertainties of typically $0.5 \%$ [1-4]. To obtain such precision results for the extraction of isolated resonance parameters, additional isospin channels and polarization as well as double-polarization observables have been measured to disentangle the individual resonant and non-resonant coupling amplitudes [4-7]. This successful effort has to be extended beyond the first and second resonance region.

Preliminary JLab [4] results at low four momentum transfers down to 0.1 $(\mathrm{GeV} / \mathrm{c})^{2}$ follow the known constant behavior for EMR and the constantly falling behavior with growing $Q^{2}$ for SMR. The extrapolation of both ratios to even smaller $\mathrm{Q}^{2}$ seem to agree with the real photon point EMR results [8] or with Siegert limit respectively, whereas the SMR disagrees with the MAMI [9] and Bates [10] results at $\mathrm{Q}^{2}=0.127(\mathrm{GeV} / \mathrm{c})^{2}$. A further extension into the momentum transfer region of $0.01(\mathrm{GeV} / \mathrm{c})^{2}<\mathrm{Q}^{2}<0.1(\mathrm{GeV} / \mathrm{c})^{2}$, where no results are available, is experimentally challenging. How strongly this lack of data impacts the comprehension of the strong interaction in this regime is illustrated in FIGURE 1. One would expect that the total cross section at $\mathrm{Q}^{2}=0(\mathrm{GeV} / \mathrm{c})^{2}$ is largest, since the $\mathrm{N}$ to $\Delta(1232)$ transition form factors should drop with increasing $\mathrm{Q}^{2}$, but the total cross section at $\mathrm{Q}^{2}=0.1 \quad(\mathrm{GeV} / \mathrm{c})^{2} \quad$ is significantly larger than at the real photon point, $Q^{2}=0$ $(\mathrm{GeV} / \mathrm{c})^{2}$ [11]. This can only be explained by a strong longitudinal coupling of the virtual photon to the nucleon and by the fact that the kinematical suppression of this

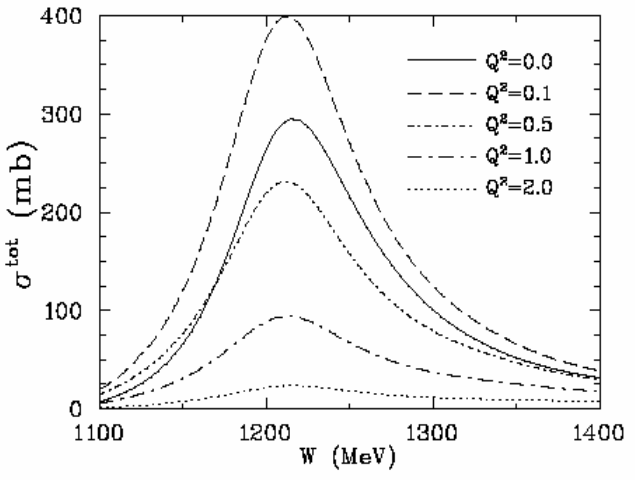

FIGURE 1: Total cross section of the $\mathrm{p} \pi^{0}$ electroproduction for different four momentum transfers in $\mathrm{GeV}\lceil 11\rceil$. longitudinal coupling drops initially much faster than the transverse transition form factors. On the other side $\mathrm{pQCD}$ predicts in the high $\mathrm{Q}^{2}$-limit, when neglecting higher twist contributions, an EMR of +1 and a $\mathrm{Q}^{2}$-independent SMR, whereas preliminary results at high $\mathrm{Q}^{2}$ up to $5(\mathrm{GeV} / \mathrm{c})^{2}$ [4] show no indication of this predicted behavior 
and follow the same overall trend as established in the non-perturbative regime. It is not only important to extend these measurements of the $\mathrm{N}$ to $\Delta(1232)$ transition form factors to even higher momentum transfers, but also to investigate the high $\mathrm{Q}^{2}$ evolution of exclusive transition form factors to other higher lying resonances. In the first case we need to push the measurements of exclusive observables and their theoretical description towards the onset of perturbative behaviour. These experiments
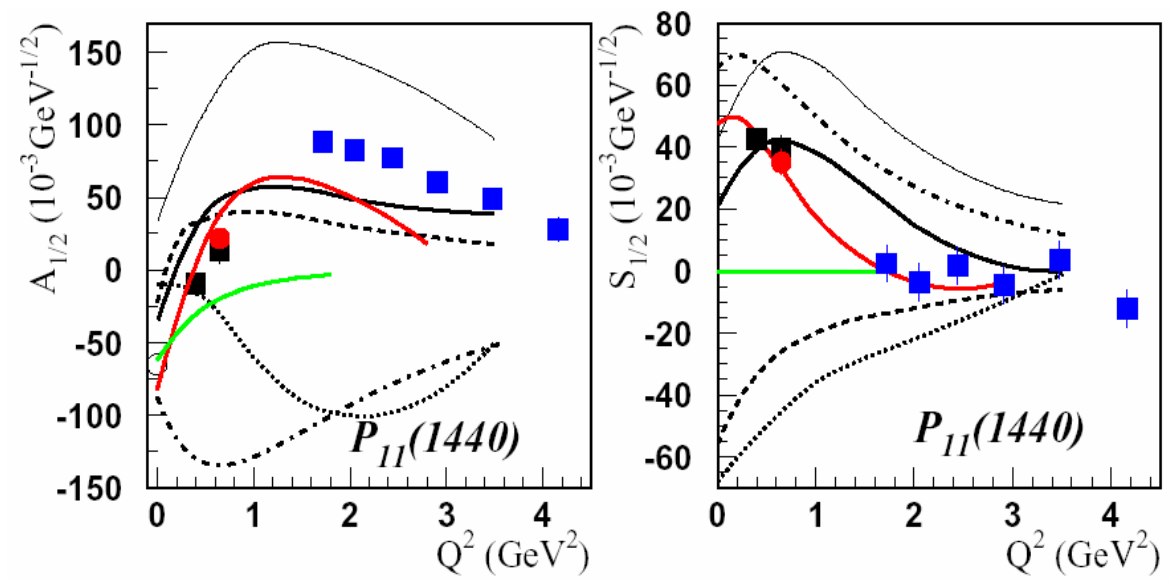

FIGURE 2: All data points are CLAS results in the $\pi$ photo-production channel (open circle) [12], $\pi$ and $2 \pi$ (solid circles) [13], and $\pi$ (solid squares) low $\mathrm{Q}^{2}[14]$ and preliminary high $\mathrm{Q}^{2}$ [15] electro-production channels. The solid bold lines are theoretical model calculations of Cano \& Gonzales: $\mathrm{qqq}+\mathrm{qq}_{\mathrm{bar}}$ cloud (red line or gray) [16], Capstick \& Keister: light-front RQM (thick black) [17], Li \& Burkert: qqqg hybrid (green line or pale gray) [18], and all other lines are further QM calculations [18,19].

require the JLab and CLAS upgrades to $12 \mathrm{GeV}$. In the latter case the $\mathrm{Q}^{2}$-dependence of the helicity coupling amplitudes for the higher lying resonances $\mathrm{P}_{11}, \mathrm{D}_{13}, \mathrm{~S}_{11}$, and $\mathrm{F}_{15}$ has been measured up to $4(\mathrm{GeV} / \mathrm{c})^{2}[15]$. FIGURE 2 illustrates the quality of the results [12-15] and the shortcomings of the model predictions [16-19]. None of the model calculations are able to describe neither the magnitude nor the $\mathrm{Q}^{2}$-trend of the helicity amplitudes of all four resonances consistently [4].

\section{MISSING RESONANCES}

The term missing resonances refers to the fact that quark models based on the flavor blindness of the strong interaction predict more nucleon excitations than are experimentally found. Besides the two commonly discussed theoretical approaches, di-quark models and coupled channel dynamics, which would reduce the number of predicted baryon states, a possible experiment-related solution to this problem is that these resonances do not couple to the single-pion nucleon channel and therefore have not been seen by now. This problem can initially be tackled from two sides by investigating both the double-pion-nucleon and the kaon-hyperon decay channels. 
Many nucleon resonances in the mass region above $1.6 \mathrm{GeV}$ decay predominantly through either $\Delta \pi$ or $\mathrm{N} \rho$ intermediate states into $\mathrm{N} \pi \pi$ final states [12]. This makes electromagnetic exclusive double-pion production an important tool in the investigation of nucleon resonances and reaction dynamics, as well as in the search for missing baryon states. Unpolarized cross-section measurements of double-pion photoand electroproduction have been reported by several groups [20] and most recently by the CLAS collaboration [21]. The $\mathrm{Q}^{2}$-dependent analysis of the $\pi^{+} \pi^{-}$electroproduction data in a phenomenological isobar model [25] indicates the presence of a new $\mathrm{P}_{13}(1720)$ resonance that couples strongly to $\Delta \pi$ and only weakly to N $\rho$. Further polarization observables are needed here as in the single-meson production to sufficiently constrain the model dependent interpretation. Polarization degrees of freedom in charged double-pion production have been investigated initially at SLAC [22] and in the context of the Gerasimov-Drell-Hearn Sum Rule at MAMI [23].

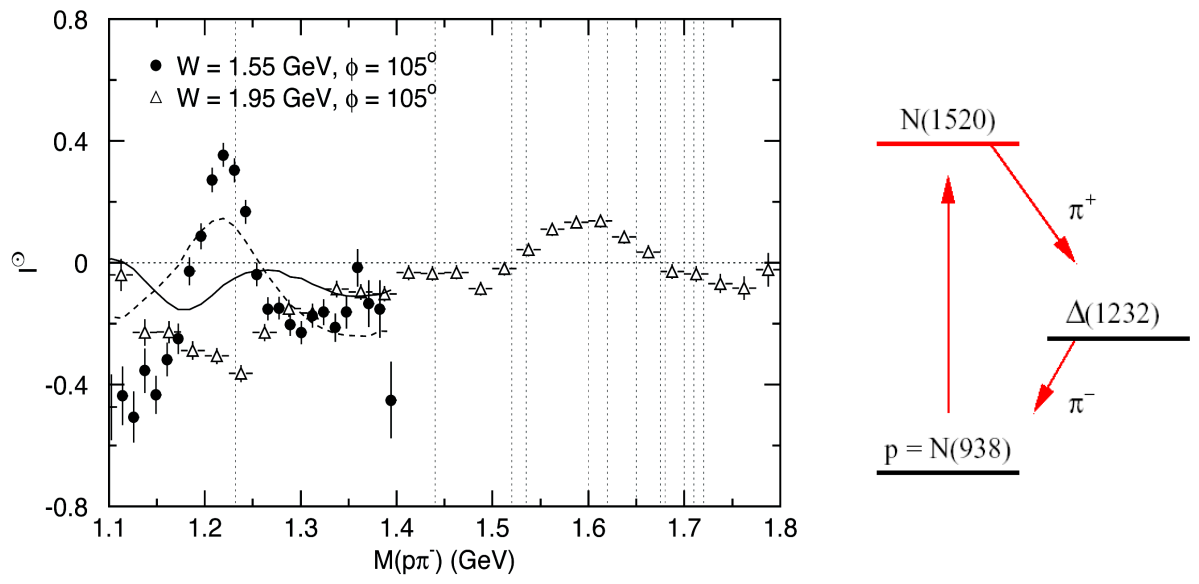

FIGURE 3: Left panel: Helicity asymmetry as a function of the invariant mass $\mathrm{M}\left(\mathrm{p} \pi^{-}\right)$for $W=$ $1.55 \mathrm{GeV}$ (filled circles) and $1.95 \mathrm{GeV}$ (open triangles) and a $30^{\circ}$-wide $\phi$-angle range centered at $\phi=105^{\circ}$ [24]. The curves are the results of Mokeev et al. [25] (solid) and Fix \& Arenhövel [26] (dashed) for $W=1.55 \mathrm{GeV}$ only. Note that the result of Fix \& Arenhövel is in phase with the data (filled circles) and that of Mokeev et al. is not. The vertical lines indicate the masses of the known $\mathrm{N}$ and $\Delta$ resonances. Right panel: Possible contributing sequential-decay channel.

New beam-helicity asymmetries have recently been reported for the double-pion photoproduction reaction $\gamma p \rightarrow p \pi^{+} \pi^{-}$[24] based on the first measurement of the helicity asymmetry $I^{0}=\left(\sigma^{+}-\sigma^{-}\right) / P_{\gamma}\left(\sigma^{+}+\sigma^{-}\right)$in the higher resonance region for center-of-mass energies between $1.35 \mathrm{GeV}$ and $2.30 \mathrm{GeV}$, where the photon beam is circularly polarized and no target nor recoil polarization is specified. Here, $\mathrm{P}_{\gamma}$ is the degree of circular polarization of the photon and $\sigma^{+}$are the cross sections for the two photonhelicity states. FIGURE 3 shows the helicity asymmetry as a function of the invariant mass $\mathrm{M}\left(\mathrm{p} \pi^{-}\right)$for two different values of $W$ and a fixed value of $\phi$ as a typical example, where $\phi$ is the angle between the scattering and the $\pi^{+} \pi^{-}$plane. The most interesting 
features are the changes that occur as $\mathrm{M}\left(\mathrm{p} \pi^{-}\right)$traverses the $\Delta(1232)$ resonance, where for $W=1.55 \mathrm{GeV}$, a maximum is seen in the region of this resonance. We see a similar trend in the region of the higher-mass resonances around $1.60 \mathrm{GeV}$ but no maximum around the $\Delta(1232)$ resonance for $W=1.95 \mathrm{GeV}$. This intriguing behavior indicates how valuable helicity asymmetry and other polarization observables can be for studying baryon spectroscopy, and how important sequential decays of resonances are in this energy range, where yet-unobserved resonances are predicted [27].

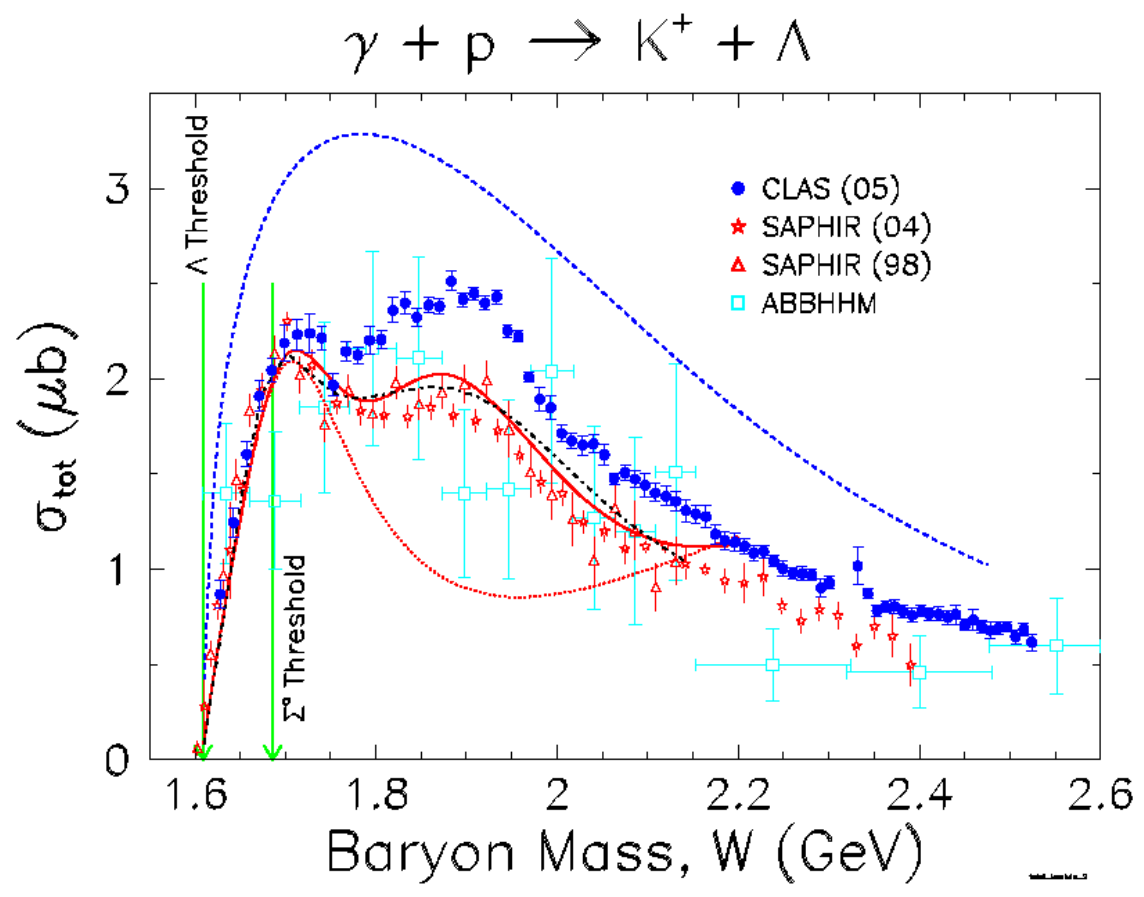

FIGURE 4: Total cross section for $\mathrm{K}^{+} \Lambda$ photoproduction. The CLAS data points (solid circles) are shown with the combined statistical and fitting uncertainties [28] and compared to the results from SAPHIR (stars [29], triangles [30]) and the ABBHHM collaboration (squares) [31]. The lines are from a Regge model (dashed) [32] Kaon-MAID [33] with (solid) and without the $\mathrm{D}_{13}(1895)$ (dotted), and an effective Lagrangian approach (dash-dotted) [34].

Very recently high-statistics photoproduction cross section data for $\mathrm{K}^{+} \Lambda$ and $\mathrm{K}^{+} \Sigma^{0}$ final states, with large kinematical coverage in both $1.6 \mathrm{GeV}<W<2.5 \mathrm{GeV}$ and $-0.85<$ $\cos \theta_{\mathrm{K}} *<0.95$, have become available [28] that allow to look for new resonances decaying into these kaon-hyperon channels. The example shown in FIGURE 4 addresses the discussion whether a new $\mathrm{D}_{13}(1895)$ as predicted by Capstick and Roberts or by Loering, Metsch and Petry [33], or a new $\mathrm{P}_{13}(1900)$ but no $\mathrm{D}_{13}(1895)$ as suggested by Shklyar, Lenske and Mosel is needed [35], or appropriate tuning of the background contributions is sufficient [34] to understand the cross section peak at 1.9 $\mathrm{GeV}$, which is now even more pronounced than previously reported by SAPHIR $[29,30]$. The additional forward-to-backward angular dependence of the $\mathrm{K}^{+} \Lambda$ cross sections reveals changes in both position and shape of this peak, which cannot be explained by a single new resonance in the s or u-channel. 


\section{ACKNOWLEDGMENTS}

We thank the staff of the Accelerator and the Physics Divisions at Thomas Jefferson National Accelerator Facility who are making experiments like these possible. Major support is provided by the Department of Energy and the National Science Foundation.

\section{REFERENCES}

1. K. Joo et al., Phys. Rev. Lett. 88, 122001 (2002) 1-5.

2. R.W. Gothe, Proceedings of NSTAR 2002, World Scientific (2003) 220-227.

3. G. Laveissiere et al., Phys. Rev. C69, 045203 (2004) 1-15.

4. Preliminary JLab Results, L.C. Smith, M. Ungaro, K. Park, Private Communication (2005).

5. K. Joo et al., Phys. Rev. C70, 042201 (2004) 1-6.

6. J.J. Kelly et al., Phys. Rev. Lett. 95102001 (2005) 1-6.

7. H. Egiyan et al., submitted to Phys. Rev. C (2005) 1-19.

8. R. Beck et al., Phys. Rev. C61, 035204 (2000) and G. Blanpied et al., Phys. Rev. Lett. 79, 4337 (1997).

9. T. Pospischil et al., Phys. Rev. Lett. 86 (2001) 2959-2962.

10. N.F. Spaveris et al., Phys. Rev. Lett. 94022003 (2005) 1-4.

11. R. Arndt et al., nucl-th/0301068 or Proceedings of NSTAR2002, Scientific (2003).

12. S. Eidelman et al., Phys. Lett. B 592, (2004) 1.

13. I.G. Aznauryan et al., Phys. Rev. C72, 045201 (2005) 1-9.

14. I.G. Aznauryan et al., Phys. Rev. C71, 015201 (2005) 1-9.

15. K. Park et al., to be submitted to Phys. Rev. Lett. (2005).

16. F. Cano and P. Gonzalez, Phys. Lett. B 431 (1998) 270-276.

17. S. Capstick and B.D. Keister, Phys. Rev. D51 (1995) 3598.

18. Z. Li and V. Burkert, Phys. Rev. D46 (1992) 70.

19. E. Pace, G. Salme and S. Simula, Few Body Syst. Suppl. 10 (1999) 407; S. Simula, Proceedings on NSTAR 2001, Mainz, Germany (2001) 135; M. Warns, H. Schroeder, W. Pfeil, and H. Rollnik, Z. Phys. C45 (1990) 627; M. Aiello et al., J. Phys. G 24 (1998) 753.

20. ABBHHM Collaboration, Phys. Rev. 175, 1669 (1968) ; ABBHHM Collaboration, Phys. Rev. 188, 2060 (1969) ; A. Braghieri et al., Phys. Lett. B363, 46 (1995); W. Langärtner et al., Phys. Rev. Lett. 87, 052001 (2001) ; M. Wolf et al., Eur. Phys. J. A9, 5 (2000) ; F. Härter et al., Phys. Lett. B401, 229 (1997) ; Y. Assafiri et al., Phys. Rev. Lett. 90, 222001 (2003).

21. M. Rapani et al., Phys. Rev. Lett. 91, 022002 (2003); S.A. Philips, Ph.D. Thesis, The George Washington University (2002); M. Bellis, Ph. D. Thesis, Rensselaer Polytechnic Institute (2004).

22. J. Ballam et al., Phys. Rev. D5, 545 (1972).

23. J. Ahrens et al., Phys. Lett. B551, 49 (2003).

24. S. Strauch et al., Phys. Rev. Lett. 95, 162003 (2005) 1-5.

25. V.I. Mokeev et al., Yad. Fiz. 64, 1368 (2001) or Phys. At. Nucl. 64, 1292 (2001).

26. A. Fix and H. Arenhövel, nucl-th/0503042 (2005).

27. S. Capstick and W. Roberts, Phys. Rev. D 49, 4570 (1994).

28. R. Bradford et al., submitted to Phys. Rev. C (2005) 1-26.

29. K.-H. Glander et al., Eur. Phys. J. A19 (2004) 251-273.

30. M.Q. Tran et al., Phys. Lett. B445, 20 (1998); M. Bockhorst et al., Z. Phys. C63, 37 (1994).

31. R. Erbe et al., Phys. Rev. 188, 2060 (1969).

32. M. Guidal et al., Nucl. Phys. A627, 645 (1997); M. Guidal et al., Phys. Rev. C61, 025204 (2000).

33. T. Mart et al. at www.kph.uni-mainz.de/MAID/kaon/kaonmaid.html.

34. B. Saghai, nucl-th/0105001 (2001).

35. V. Shklyar et al., Phys. Rev. C72, 015210 (2005) 1-8. 